\title{
Estimation of physical transformer parameters from frequency response analysis
}

by Steven Douglas Mitchell, James Stuart Welsh

Copyright (C) 2011 IEEE.

This is an author-prepared version of the article, reprinted from Proceedings of the IEEE PES Trondheim PowerTech 2011: The Power of Technology for a Sustainable Society.

http://dx.doi.org/10.1109/PTC.2011.6019187

This material is posted here with permission of the IEEE. Such permission of the IEEE does not in any way imply IEEE endorsement of any of University of Newcastle's products or services. Internal or personal use of this material is permitted. However, permission to reprint/republish this material for advertising or promotional purposes or for creating new collective works for resale or redistribution must be obtained from the IEEE by writing to pubs-permissions@ieee.org. By choosing to view this document, you agree to all provisions of the copyright laws protecting it. 


\section{Estimation of Physical Transformer Parameters from Frequency Response Analysis}

\author{
Steven D. Mitchell \\ School of Electrical and \\ Computer Engineering \\ University of Newcastle \\ Callaghan, Australia 2308 \\ Email: steve.mitchell@newcastle.edu.au
}

\author{
James S. Welsh \\ School of Electrical and \\ Computer Engineering \\ University of Newcastle \\ Callaghan, Australia 2308 \\ Email: james.welsh@newcastle.edu.au
}

\begin{abstract}
The wideband frequency response of a power transformer will yield a signature unique to its mechanical geometry. A change in the frequency response can be indicative of winding deformation. Frequency Response Analysis (FRA) is a diagnostic tool used to monitor frequency response changes. The aim of this paper is to facilitate FRA interpretation through the development of a three phase transformer model that is based on the estimation of physical parameters. The paper will demonstrate the applicability of the model by using FRA data sets of a 1.3MVA distribution transformer and assessing the accuracy of key parameter estimates.
\end{abstract}

\section{INTRODUCTION}

Due to infrastructure expansion and the high replacement costs associated with a power transformer, the average age of power transformers is increasing with many exceeding their nominal life expectancy [1]. As a direct result, research into maximizing their longevity is a high priority. To successfully accomplish this in a safe and efficient manner, their condition must be regularly monitored in order to schedule the appropriate maintenance and repair. Fault currents in a power transformer subject the windings and associated mechanical structure to high levels of mechanical stress. This stress can lead to winding deformation and hence, potentially, to transformer failure.

Winding deformation will result in subtle changes to the inductive and capacitive relationships of the winding. Frequency Response Analysis (FRA) is a commonly used tool for monitoring the winding deformation of a power transformer. It is well known that the wideband frequency response of a transformer will yield a signature unique to its mechanical geometry [2]. Generally, it is industry practice for trained personnel to visually compare the frequency response of different phases in the same apparatus, or the same phase on sister units. Variation of the comparative responses indicates a geometric change and can be indicative of structural damage. However, there is no understanding of the actual underlying cause of the change.

CIGRE's WG A2.26 working group has emphasized the need for FRA modeling to support interpretation of a measured FRA response [3]. The aim of this paper is to assist in facilitating FRA interpretation by presenting a three phase lumped parameter transformer model that is founded on physically measurable parameters. The paper will demonstrate the model's applicability by fitting the model to the FRA data sets of a $1.3 \mathrm{MVA} 11 \mathrm{kV} / 430 \mathrm{~V}$ distribution transformer and quantitatively assessing each of the estimated parameter's accuracy.

Generic phase referencing has been implemented throughout this paper to accommodate for the various phase permutations involved in FRA testing. The three generic high voltage terminals are $\mathrm{X}-\mathrm{Y}-\mathrm{Z}$, and the corresponding low voltage terminals are $\mathrm{x}-\mathrm{y}-\mathrm{Z}$.

\section{Generic Phase Model}

The proposed $n$ section lumped parameter transformer model for generic phase $X$ is presented in Figure 1. Each section of the high and low voltage windings consists of the series combination of an inductive element $\mathfrak{L}$ and a resistive element $\mathfrak{R}$. To account for the capacitance between these windings, a capacitive element $\mathfrak{C}_{X x}$ couples each equivalent winding section. The capacitance between turns and adjacent discs is modelled through the addition of $\mathfrak{C}_{S X}$ and $\mathfrak{C}_{S x}$, for the high and low voltage windings respectively. The capacitance between the low voltage winding and ground is given by $\mathfrak{C}_{g x}$ and the capacitance between the high voltage winding and the transformer tank wall/s is given by $\mathfrak{C}_{g X}$. Finally, the capacitances $\mathfrak{C}_{X Y}$ and $\mathfrak{C}_{Z X}$ represent the capacitance between adjacent high voltage windings (i.e. A to B and B to C). Note that the magnitude of all shunt capacitances at the winding ends are at half their normal value to reflect their relative distribution.

Each of the inductive, resistive and capacitive elements shown in Figure 1 will now be discussed in detail.

\section{A. Inductive Element}

The use of a reluctance model is a convenient method for modelling flux paths within a transformer. This modelling approach is a physical representation, utilising a magnetic circuit based on the transformer's core geometry. In the magnetic circuit, each winding is replaced with a magnetomotive source and each flux path, including those representative of leakage, with a corresponding reluctance. In this paper we assume that the transformer is of core type construction with concentric 


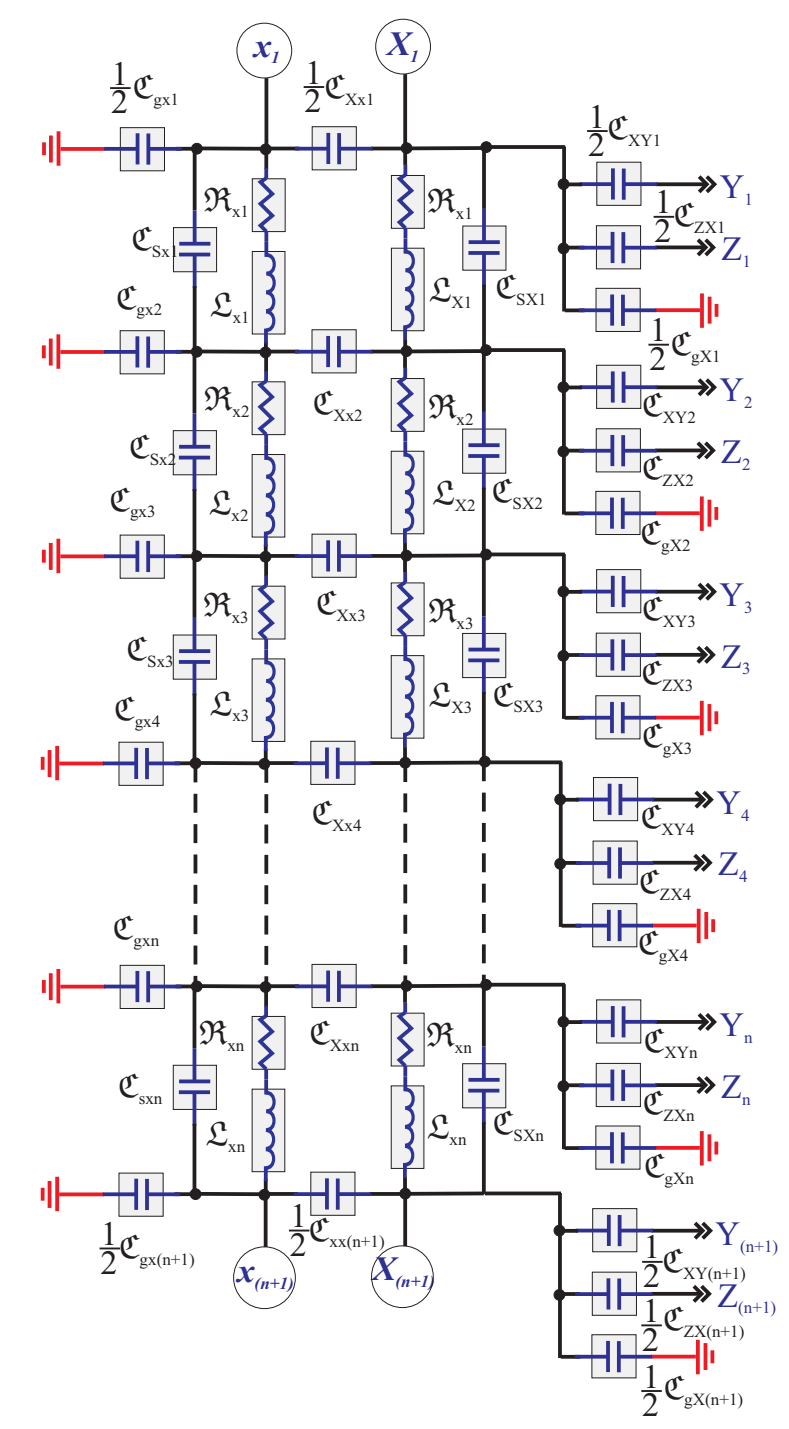

Figure 1. Lumped parameter transformer model for generic phase $\mathrm{X}$

windings. The leakage inductance is considered to be restricted to the axial path between the high and low voltage windings [4].

The proposed magnetic circuit for an FRA injection test is presented in Figure 2. In this figure, $\mathbb{F}_{\mathrm{A}}$ represents the magnetomotive force $(\mathrm{mmf})$ due to the high voltage winding of phase $A, \mathbb{F}_{\mathrm{a}}$ the mmf due to the low voltage winding on phase $\mathrm{A}$ and so forth for the other phases. $\mathbb{R}_{\mathrm{E}}$ is the transformer core limb reluctance and $\mathbb{R}_{Y}$ is the transformer core yoke reluctance. $\mathbb{R}_{\mathrm{L}}$ is the winding leakage flux reluctance. The lineal dimensions of the core are $l_{E}$ for the mean core limb length and $l_{W}$ for the mean core yoke width.

The core reluctance can be defined in terms of the mean path length $l$, core cross sectional area $A_{C S}$, and the core

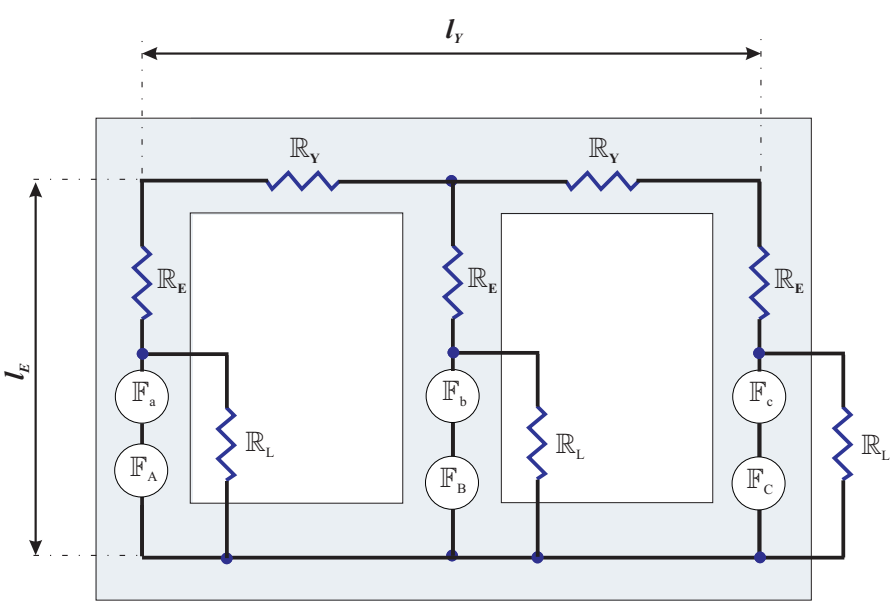

Figure 2. Magnetic equivalent circuit of a three phase two winding core type transformer

permeability $\mu$ such that,

$$
\begin{aligned}
\mathbb{R}_{E} & =\left[\frac{1}{\mu A_{C S}}\right] l_{E} \\
\mathbb{R}_{\mathrm{Y}} & =\left[\frac{1}{\mu A_{C S}}\right] l_{Y},
\end{aligned}
$$

where $\mathbb{R}_{\mathrm{E}}$ and $\mathbb{R}_{\mathrm{Y}}$ are directly proportional to the limb and yoke length. By definition, inductance is proportional to a winding's flux linkage relative to the current in the winding for self inductance, or the current in another winding for mutual inductance. From (1) and (2), the inductive relationships that exist between winding sections on the three phase transformer core will therefore be dependent upon the core dimensions. By defining a base inductance to be,

$$
\bar{L}=\frac{\mu A_{C S}\left(\frac{N_{X}}{n}\right)^{2}\left(2 l_{E}+l_{Y}\right)}{\left(l_{E}+l_{Y}\right)\left(3 l_{E}+l_{Y}\right)}
$$

and two core dimension constants to be,

$$
\begin{gathered}
\Gamma=\frac{2 l_{E}+l_{Y}}{2\left(l_{E}+l_{Y}\right)} \\
\Lambda=\frac{2 l_{E}+l_{Y}}{l_{E}},
\end{gathered}
$$

where $n$ is the number of lumped parameter sections within the transformer model, a matrix that represents each of the respective sectional inductances can be generated. This matrix is presented in Table I where $i$ and $j$ are the lumped parameter section numbers and $\hat{a}$ is the turns ratio.

The model also incorporates a generic leakage inductance between winding sections. For example, the leakage inductance between sections $i$ and $j$ of generic winding $X$ is given by $L_{L X i j}$.

The permeability of the transformer core as specified in the base inductance relationship of (3) is a complex frequency dependent term. Under the low field conditions and wide 


\begin{tabular}{|c|c|c|c|c|c|c|}
\hline$L$ & $A_{j}$ & $B_{j}$ & $C_{j}$ & $a_{j}$ & $b_{j}$ & $c_{j}$ \\
\hline \hline$A_{i}$ & $\bar{L}$ & $\frac{\bar{L}}{2 \Gamma}$ & $\frac{\bar{L}}{\Lambda}$ & $\frac{\bar{L}}{\hat{a}}$ & $\frac{\bar{L}}{2 \Gamma \hat{a}}$ & $\frac{\bar{L}}{\Lambda \hat{a}}$ \\
\hline$B_{i}$ & $\frac{\bar{L}}{2 \Gamma}$ & $\frac{\bar{L}}{\Gamma}$ & $\frac{\bar{L}}{2 \Gamma}$ & $\frac{\bar{L}}{2 \Gamma \hat{a}}$ & $\frac{\bar{L}}{\Gamma \hat{a}}$ & $\frac{\bar{L}}{2 \Gamma \hat{a}}$ \\
\hline$C_{i}$ & $\overline{\bar{L}}$ & $\frac{\bar{L}}{2 \Gamma}$ & $\bar{L}$ & $\frac{\bar{L}}{\Lambda \hat{a}}$ & $\frac{\bar{L}}{2 \Gamma \hat{a}}$ & $\frac{\bar{L}}{\hat{a}}$ \\
\hline$a_{i}$ & $\frac{\bar{L}}{\hat{a}}$ & $\frac{\bar{L}}{2 \Gamma \hat{a}}$ & $\frac{\bar{L}}{\Lambda \hat{a}}$ & $\frac{\bar{L}}{\hat{a}^{2}}$ & $\frac{\bar{L}}{2 \Gamma \hat{a}^{2}}$ & $\frac{\bar{L}}{\Lambda \hat{a}^{2}}$ \\
\hline$b_{i}$ & $\frac{\bar{L}}{2 \Gamma \hat{a}}$ & $\frac{\bar{L}}{\Gamma \hat{a}}$ & $\frac{\bar{L}}{2 \Gamma \hat{a}}$ & $\frac{\bar{L}}{2 \Gamma \hat{a}^{2}}$ & $\frac{\bar{L}}{\Gamma \hat{a}^{2}}$ & $\frac{\bar{L}}{2 \Gamma \hat{a}^{2}}$ \\
\hline$c_{i}$ & $\frac{\bar{L}}{\Lambda \hat{a}}$ & $\frac{\bar{L}}{2 \Gamma \hat{a}}$ & $\frac{\bar{L}}{\hat{a}}$ & $\frac{\bar{L}}{\Lambda \hat{a}^{2}}$ & $\frac{\bar{L}}{2 \Gamma \hat{a}^{2}}$ & $\frac{\frac{\bar{L}}{\hat{a}^{2}}}{}$ \\
\hline
\end{tabular}

Table I

LUMPED PARAMETER TRANSFORMER INDUCTANCE MATRIX

centre, which results in an increase in the effective resistance. An analytical estimate for skin effect can be made using the Dowell Method [7]. If it is assumed that the conductors are closely packed, each layer of a winding will approximate the geometry of a conductor foil. The problem can then be reduced to a one dimensional model where [8],

$$
R_{S}=\frac{R_{D C} \xi}{2}\left[\frac{\sinh \xi+\sin \xi}{\cosh \xi-\cos \xi}\right]
$$

where

$$
\xi=\frac{d \sqrt{\pi}}{2 \delta}
$$

frequency spectrum that would be observed during an FRA test, $\mu$ can be defined to be [5],

$$
\mu=\mu_{0} \mu_{s}=\mu_{0}\left(\mu_{s}^{\prime}-j \mu_{s}^{\prime \prime}\right)=\frac{k \mu_{0} \mu_{i}}{\gamma b} \tanh (\gamma b) .
$$

In this relationship $\mu_{0}$ is the permeability of free space, $\mu_{s}$ is the effective permeability, $u_{i}$ is the initial permeability of the core material, $k$ is the lamination stacking factor (which approaches unity) and $b$ is the core lamination half thickness. The propagation constant, $\gamma$, is defined as,

$$
\gamma=\sqrt{j \omega \sigma \mu_{0} \mu_{i}},
$$

where $w$ is the angular frequency in radians per second, and $\sigma$ is the lamination material conductivity. As a result of the complex permeability of the core in (6), a winding's inductance and its associated magnetic losses can be represented as an impedance. This impedance can be modelled as the series combination of a frequency dependent inductance, $L^{\prime}$, and resistance $R^{\prime \prime}$ [6],

$$
\begin{aligned}
Z & =j w L_{0}\left(\mu_{s}^{\prime}-j \mu_{s}^{\prime \prime}\right) \\
& =j w L^{\prime}+R^{\prime \prime},
\end{aligned}
$$

where $R^{\prime \prime}$ can be considered to be the magnetic loss resistance and $L_{0}$ is the inductance with unity permeability.

An inductive element $\mathfrak{L}_{X i}(\omega)$ is now proposed that takes into account the self and mutual inductance relationships described in Table I and the real and imaginary components of (9). $\mathfrak{L}_{X i}(\omega)$ represents the frequency dependent inductive contribution of section $i$ of generic winding $X$ and is presented in Figure 3(a).

\section{B. Resistive Element}

The transformer winding can be considered to have an inherent DC resistance loss term and a frequency dependent AC resistance loss term. The DC loss term is directly proportional to the resistivity of the conductor and inversely proportional to the winding conductor cross sectional area. The $\mathrm{AC}$ resistance is due to the induction of eddy currents within the windings. These induced resistive losses can be classified into two categories, skin and proximity effect.

Skin effect is due to the magnetic field generated by the current in the conductor. This has the effect of increasing the current density near the conductor surface relative to its

$d$ is the conductor diameter and

$$
\delta=\frac{1}{\sqrt{\pi f \mu \sigma}},
$$

is the skin depth, with $f$ the frequency in $\mathrm{Hz}$, and the permeability and conductivity of the conductor material are given by $\mu$ and $\sigma$ respectively.

Proximity effect is where the current in adjacent conductors generate magnetic fields which induce circulating eddy currents in neighbouring conductors. This will impact on the conductors current distribution, and therefore will also increase the effective resistance. Assuming once again that the conductors are closely packed and that each layer of a winding will approximate the geometry of a conductor foil, the AC resistance due to proximity effect for the $m$ th winding layer [8] is,

$$
R_{P}=\frac{R_{D C} \xi}{2}\left[(2 m-1)^{2} \frac{\sinh \xi-\sin \xi}{\cosh \xi+\cos \xi}\right] .
$$

Assuming that the magnetic field from the other conductors is uniform across the conductor cross section, an orthogonal relationship exists between the skin and proximity effect [9]. The two effects can be decoupled and an estimate for the total eddy current losses can be determined through the addition of both effects (10) and (13). By then combining both the AC and DC winding loss contributions and considering them to be sectionally distributed, the wideband series resistance element, $\mathfrak{R}_{X i}$, is given by,

$$
\mathfrak{R}_{X i}(\omega)=R_{P X i}(\omega)+R_{S X i}(\omega)+R_{D C X i},
$$

and is presented in Figure 3(c).

\section{Capacitive Element}

In addition to a capacitor's displacement current, a dielectric material will also experience losses through conduction and material polarization [10]. For the non-ideal capacitance $\mathfrak{C}$, the circuit admittance is given by [10] [11],

$$
\begin{aligned}
Y & =j \omega \mathfrak{C} \\
& =G+j \omega C .
\end{aligned}
$$

It is well known that the admittance of a non-ideal capacitance can be represented as the parallel combination of a loss conductance $G$ and an ideal capacitance $C$ [4]. The lumped 


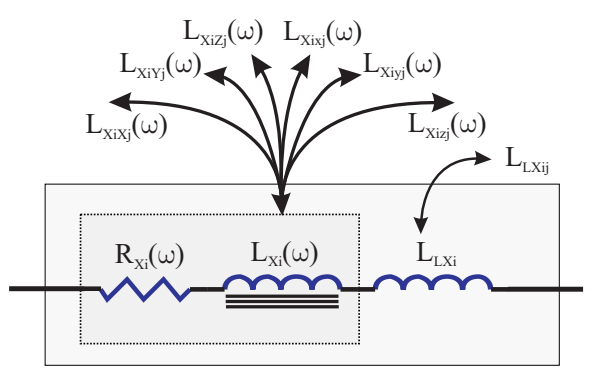

(a)

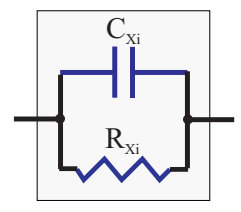

(b)

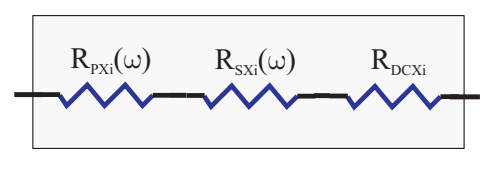

(c)
Figure 3. Lumped parameter circuit elements. (a) $\mathfrak{L}_{X i}(\omega)$, (b) $\mathfrak{C}_{X i}(\omega)$, (c) $\mathfrak{R}_{X i}(\omega)$

parameter circuit element used to represent each of the nonideal capacitors used in the transformer model takes the form presented in Figure 3(b). For mathematical convenience an equivalent resistance is substituted for conductance.

\section{MODELling FOR FRA}

There are a number of different FRA test types. These include end to end open circuit, end to end short circuit, capacitive interwinding and inductive interwinding tests [12]. For the sake of clarity we focus on the high voltage winding end to end open circuit test on a Dyn connected transformer. This test involves injecting a swept frequency sine wave into one of the high voltage terminals and recording the output response on another. All of the remaining terminals are left unconnected. This procedure is conducted on each of the three terminal pair permutations.

To facilitate our FRA modelling, the input terminal for this test sequence is labelled generic terminal $X$ and the output terminal generic terminal $\mathrm{Y}$ (terminals $\mathrm{Z}, \mathrm{x}, \mathrm{y}$ and $\mathrm{Z}$ are unconnected). The modelling approach involves the substitution of the physical phases, A through $\mathrm{C}$, into a prescribed generic counterpart, $\mathrm{X}$ through $\mathrm{Z}$, for each of the three FRA test combinations ( $\mathrm{AB}, \mathrm{BC}$ and $\mathrm{CA})$. The generic approach also facilitates modelling the phase order differences between Dyn1 and Dyn11 connections. Note that the winding with a direct connection between the FRA input and output terminals will always be winding $\mathrm{X}$. This is important due to the subtle disparity in inductance between a phase $\mathrm{B}$ winding and that of phases A or C. Because of this disparity, the low frequency response is dependent upon the FRA test pair and the transformer's vector group [13].

In order to develop a flexible FRA model that can be expanded to take into account the different FRA tests and transformer vector group connections, a layered modelling approach has been adopted. In this approach the first layer is the generic phase model presented in Figure 1. The layer above this is the vector group topology of the transformer, which is Dyn in this case. The outermost layer is the end to end FRA test connection. The modelling layer approach is presented in Figure 4.

\section{MAThematical Model}

\section{A. Normal Tree Conversion}

To conduct analysis on a complex circuit design such as the model structures that are proposed, it is advantageous to convert the proposed model into its normal tree form [14]. The normal tree associated with the layered model of Figure 4 is presented in Figure 5.

\section{B. State Space Equation}

For system analysis it is convenient to use a state space representation. The state space equations for a network tree [14] are given by,

$$
\left[\begin{array}{c}
\dot{\boldsymbol{q}}(t) \\
\dot{\boldsymbol{\phi}}(t)
\end{array}\right]=\mathbf{A}\left[\begin{array}{c}
\boldsymbol{q}(t) \\
\boldsymbol{\phi}(t)
\end{array}\right]+\mathbf{B v}_{V}(t)
$$

where

$$
\begin{aligned}
& \mathbf{A}=-\left[\begin{array}{cc}
\mathbb{K}_{C C} \mathbb{C}^{-1} & \mathbb{K}_{C \mathfrak{L}} \mathfrak{L}_{M}^{-1} \\
\mathbb{K}_{\mathfrak{L} C} \mathbb{C}^{-1} & \mathbb{K}_{\mathfrak{L} \mathfrak{L}} \mathfrak{L}_{M}^{-1}
\end{array}\right], \\
& \mathbf{B}=\left[\begin{array}{c}
\mathbb{K}_{C C} \mathbb{C}^{-1} \mathbf{F}_{C S} \mathbf{C}_{S} \mathbf{F}_{V S}^{T}-\mathbb{K}_{C V} \\
\mathbb{K}_{\mathfrak{L} C} \mathbb{C}^{-1} \mathbf{F}_{C S} \mathbf{C}_{S} \mathbf{F}_{V S}^{T}-\mathbb{K}_{V \mathfrak{L}}
\end{array}\right] .
\end{aligned}
$$

The state variable $\boldsymbol{q}(t)$ is defined as,

$$
\boldsymbol{q}(t)=\mathbb{C v}_{C}(t)+\mathbf{F}_{C S} \mathbf{C}_{S} \mathbf{F}_{V S}^{T} \mathbf{v}_{V}(t),
$$

which represents the "net-charge-per-capacitance-treebranch-cutset". The state variable $\phi(t)$ is defined to be,

$$
\phi(t)=\mathfrak{L}_{M} \mathbf{i}_{\mathfrak{L}}(t),
$$

which represents the "net-flux-per-inductance-linkfundamental-loop".

\section{Transfer Function}

An FRA test results in a plot of the frequency response of the relationship between the recorded output voltage and the injected input voltage. This is effectively the transfer function between the two FRA test terminals of the transformer. With reference to the normal tree model of the FRA test in Figure 5 , the FRA input voltage is given by $V_{I N}$ and the FRA output voltage is equivalent to the voltage drop across the $50 \Omega$ termination resistor $R_{T}$. This voltage drop can be considered in terms of branch capacitor voltage drops. From Figure 5, the output voltage is equivalent to the combined voltage drops across the branch capacitors $C_{g y 1}$ and $C_{Y y 1}$. The transfer function can then be determined from the resulting input and output voltage relationships.

The capacitor branch voltages can be determined from the state space equation of (16) in the following manner. First, take the Laplace transform of the state space relationship,

$$
\left[\begin{array}{l}
\boldsymbol{q}(s) \\
\boldsymbol{\phi}(s)
\end{array}\right]=(\mathbf{s I}-\mathbf{A})^{-1} \mathbf{B v}_{V}(s) \text {. }
$$




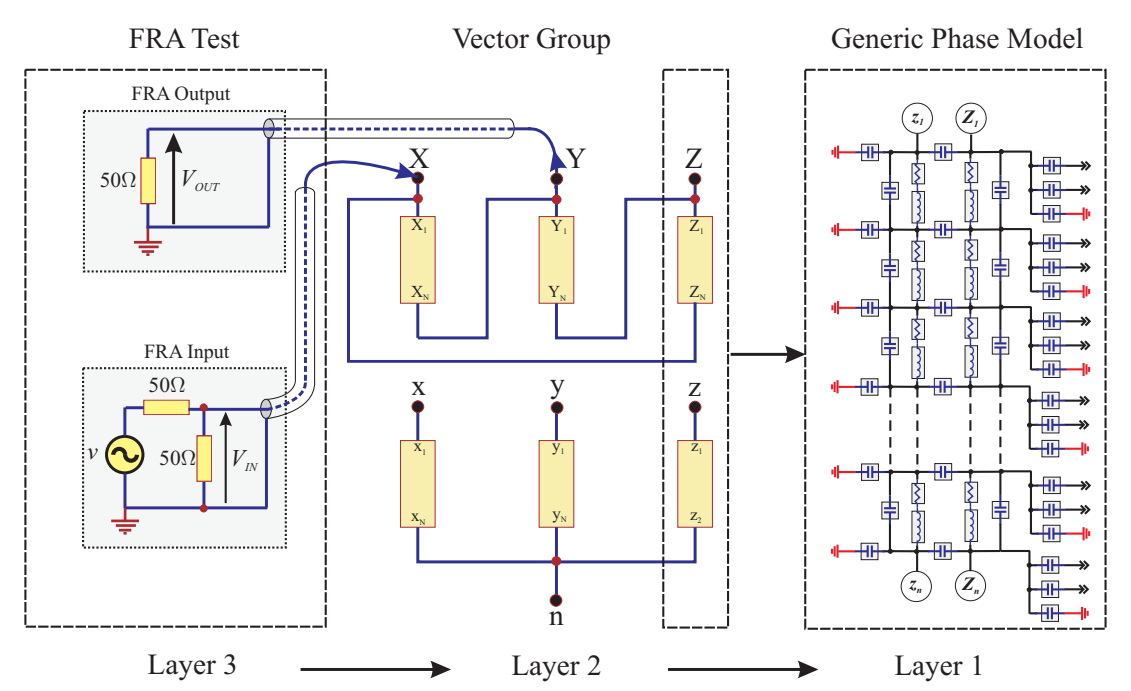

Figure 4. Layered modelling approach.

$\boldsymbol{q}(s)$ and $\phi(t)$ can be written in terms of their matrix elements,

$$
\begin{aligned}
\boldsymbol{q}(s) & =\left[\begin{array}{lll}
q(s)_{1} & \cdots & q(s)_{6 n}
\end{array}\right]^{T} . \\
\phi(s) & =\left[\begin{array}{lll}
\phi(s)_{1} & \cdots & \phi(s)_{6 n}
\end{array}\right]^{T} .
\end{aligned}
$$

With $\mathbf{v}_{V}(s)=V_{I N}(s)$, we can then define a matrix $\mathbf{P}$ such that,

$$
\mathbf{P}=(\mathbf{s} \mathbf{I}-\mathbf{A})^{-1} \mathbf{B}=\left[p_{1} \cdots p_{12 n}\right]^{T}
$$

The relationship in (24) can be written in block form relative to $\boldsymbol{q}(s)$ and $\boldsymbol{\phi}(s)$,

$$
\mathbf{P}=\left[\mathbf{P}_{q} \vdots \mathbf{P}_{\phi}\right]^{T},
$$

where

$$
\mathbf{P}_{q}=\left[\begin{array}{lll}
p_{1} & \cdots & p_{6 n}
\end{array}\right]
$$

and

$$
\mathbf{P}_{\phi}=\left[\begin{array}{lll}
p_{(6 n+1)} & \cdots & p_{12 n}
\end{array}\right] .
$$

Hence,

$$
\boldsymbol{q}(s)=\mathbf{P}_{q}^{T} V_{I N}(s),
$$

and,

$$
\phi(s)=\mathbf{P}_{\phi}^{T} V_{I N}(s) .
$$

Equating (19) with (28) and substituting $V_{I N}(s)$ for $\mathbf{v}_{V}(s)$,

$$
\begin{aligned}
\mathbf{P}_{q}^{T} V_{I N}(s) & =\mathbb{C v}_{C}(s)+\mathbf{F}_{C S} \mathbf{C}_{S} \mathbf{F}_{V S}^{T} V_{I N}(s) \\
\mathbb{C v}_{C}(s) & =\mathbf{P}_{q}^{T} V_{I N}(s)-\mathbf{F}_{C S} \mathbf{C}_{S} \mathbf{F}_{V S}^{T} V_{I N}(s) \\
\mathbf{v}_{C}(s) & =\mathbb{C}^{-1}\left[\mathbf{P}_{q}^{T}-\mathbf{F}_{C S} \mathbf{C}_{S} \mathbf{F}_{V S}^{T}\right] V_{I N}(s)
\end{aligned}
$$

The relationship defined in (30) facilitates the determination of all of the capacitance branch voltages since,

$$
\begin{aligned}
& \mathbf{v}_{C}(s)=\left[\begin{array}{llllll}
v_{C g x 2} & \cdots & v_{C g x(n+1)} & v_{C g y 1} & \cdots & v_{C g y(n)}
\end{array}\right. \\
& \begin{array}{llllll}
v_{C g z 1} & \cdots & v_{C g z(n)} & v_{C X x 1} & \cdots & v_{C X x(n)}
\end{array} \\
& \left.\begin{array}{llllll}
v_{C Y y 1} & \cdots & v_{C Y y(n)} & v_{C Z z 1} & \cdots & V_{C Z z(n)}
\end{array}\right]_{6 n \times 1}^{T}(31)
\end{aligned}
$$

To determine $V_{O U T}(s)$ we will define a matrix $\mathbf{W}$ of dimension $1 \times 6 n$ for the summation of the appropriate branch capacitor voltages such that,

$$
V_{O U T}(s)=\mathbf{W}_{\mathbf{v}_{\mathbf{C}}}(s) .
$$

In this relationship, the matrix $\mathbf{W}$ is used to sum the respective branch capacitors of $\mathbf{v}_{C}(s)$ (31). For the high voltage end to end FRA test of Figure 5, $V_{O U T}$ is the combined voltage drops across the branch capacitors $C_{g y 1}$ and $C_{Y y 1}$, and hence,

$$
\begin{aligned}
& \mathbf{W}=\left[\begin{array}{lllllll}
0_{1} & \cdots & 0_{n} & 1_{(n+1)} & 0_{(n+2)} & \cdots & 0_{4 n}
\end{array}\right. \\
& \left.1_{(4 n+1)} 0_{(4 n+2)} \cdots 0_{6 n}\right]_{1 \times 6 n} .
\end{aligned}
$$

Substituting (30) into (32),

$$
\begin{aligned}
V_{O U T}(s) & =\mathbf{W} \mathbb{C}^{-\mathbf{1}}\left[\mathbf{P}_{\mathbf{q}}^{\mathbf{T}}-\mathbf{F}_{\mathbf{C S}} \mathbf{C}_{\mathbf{S}} \mathbf{F}_{\mathbf{V S}}^{\mathbf{T}}\right] V_{I N}(s) \\
\therefore \hat{H}(s) & =\frac{V_{O U T}(s)}{V_{I N}(s)} \\
& =\mathbf{W} \mathbb{C}^{-\mathbf{1}}\left[\mathbf{P}_{\mathbf{q}}^{\mathbf{T}}-\mathbf{F}_{\mathbf{C S}} \mathbf{C}_{\mathbf{S}} \mathbf{F}_{\mathbf{V S}}^{\mathbf{T}}\right]
\end{aligned}
$$

\section{Estimation Algorithm}

A gradient search based estimation algorithm is applied to determine the transfer function parameters of (34) with respect to the recorded FRA data. This algorithm determines the best fit between the proposed model and the FRA data by finding the model parameters that result in the lowest cost. The cost, $J$, is calculated using the cumulative residual between corresponding model and data frequency points. Since a generic 


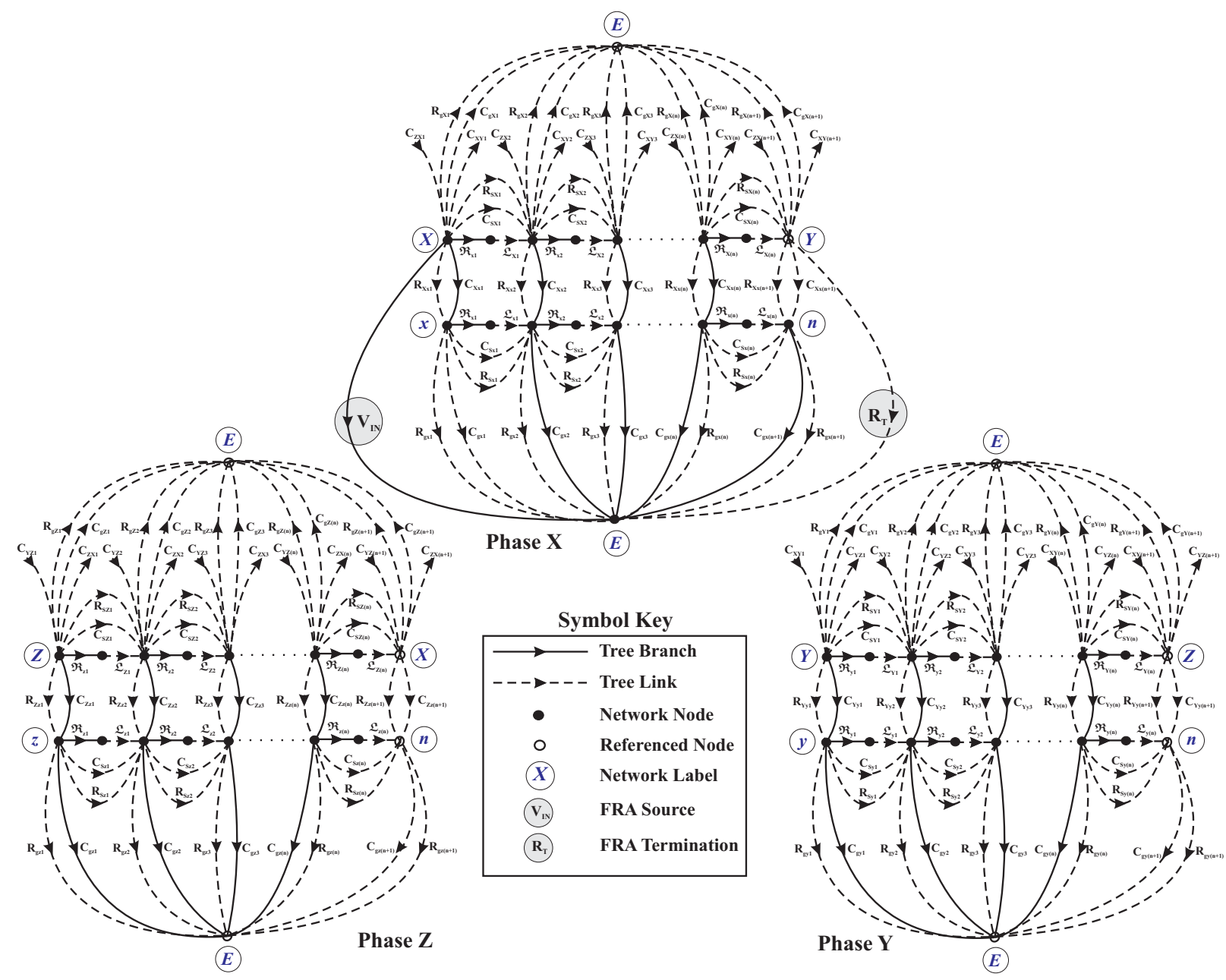

Figure 5. Normal tree representation for a high voltage winding end to end open circuit FRA test on a generic Dyn connected transformer model.

model is applied to each of the three phase permutations of the FRA test (A to $\mathrm{C}, \mathrm{B}$ to $\mathrm{A}$ and $\mathrm{C}$ to $\mathrm{B}$ ), then $J$ is the combined cost of each test,

$$
\begin{aligned}
J= & \left\|\log _{10}\left(\frac{\hat{H}_{A C}(s)}{H_{A C}(s)}\right)\right\|^{2}+ \\
& \left\|\log _{10}\left(\frac{\hat{H}_{B A}(s)}{H_{B A}(s)}\right)\right\|^{2}+ \\
& \left\|\log _{10}\left(\frac{\hat{H}_{C B}(s)}{H_{C B}(s)}\right)\right\|^{2},
\end{aligned}
$$

where $H(s)$ is the FRA data and $\hat{H}(s)$ is the model transfer function.

\section{RESUlTS}

The estimation algorithm is applied to determine the parameters of the model transfer function (34) for the high voltage end to end open circuit FRA test data of a 1.3MVA $11 \mathrm{kV} / 430 \mathrm{~V}$ Dyn1 distribution transformer. The algorithm is simultaneously applied to the FRA data sets of all three phase

\begin{tabular}{|l|c|c|c|}
\hline Parameter & Actual & Estimate & Error \\
\hline \hline HV winding turns & 836 & 901 & $+8 \%$ \\
\hline Core cross sectional area $\left(\mathrm{m}^{2}\right)$ & 0.033 & 0.025 & $-23 \%$ \\
\hline Core yoke length $(\mathrm{m})$ & 1.09 & 1.02 & $-6 \%$ \\
\hline Core limb length $(\mathrm{m})$ & 1.24 & 0.93 & $-25 \%$ \\
\hline
\end{tabular}

Table II

COMPARISON BETWEEN ESTIMATED MODEL PARAMETERS AND THEIR ACTUAL VALUES.

permutations (AC, BA and $\mathrm{CB}$ ). The results are presented in Figures 6, 7 and 8. As observed in these figures, the results in both magnitude and phase are quite good.

To demonstrate the physical applicability of the model, parameters which are readily measurable are compared with their estimated counterparts in Table II. Whilst the relative errors are not insignificant, when it is considered that these parameters have been determined from FRA data, the results are very encouraging. 

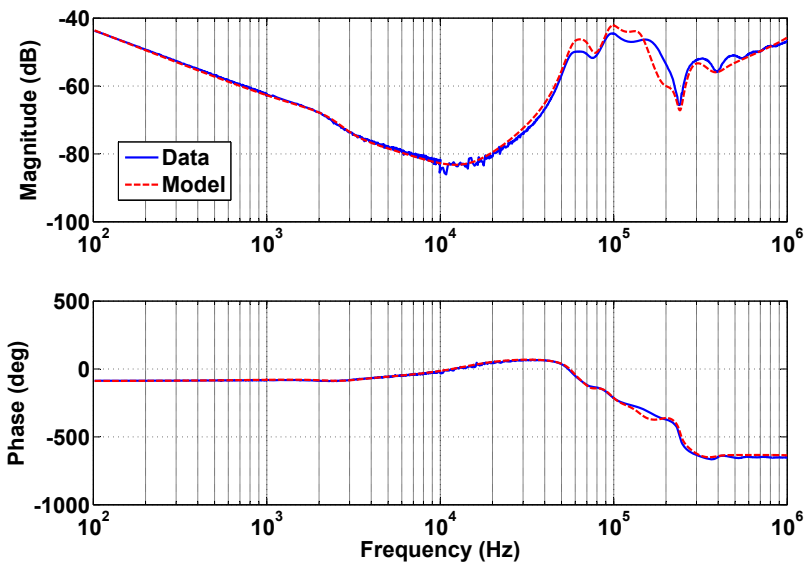

Figure 6. Estimated model and FRA data measured between the high voltage $\mathrm{A}$ and $\mathrm{C}$ terminals on a $1.3 \mathrm{MVA} 11 \mathrm{kV} / 430 \mathrm{~V}$ Dyn 1 transformer.
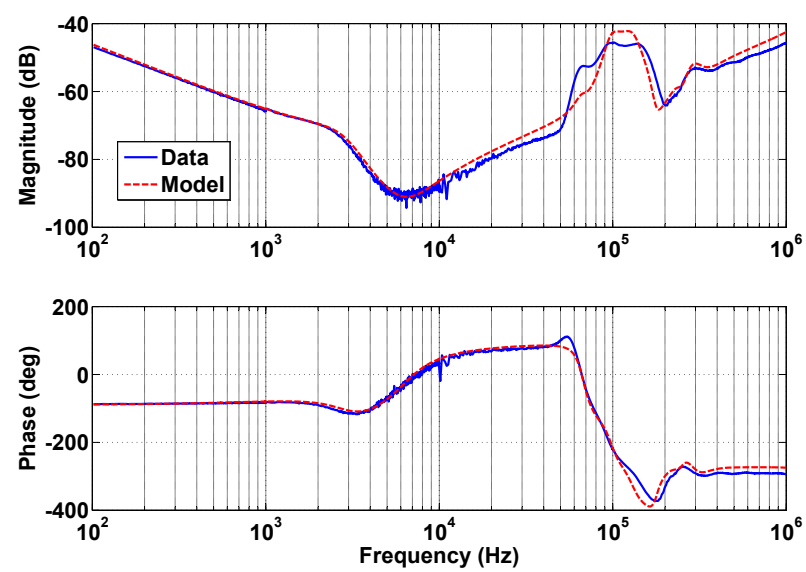

Figure 7. Estimated model and FRA data measured between the high voltage $\mathrm{B}$ and A terminals on a $1.3 \mathrm{MVA} 11 \mathrm{kV} / 430 \mathrm{~V}$ Dyn 1 transformer.

\section{CONCLUSION}

The CIGRE working group A2.26 has emphasised how transformer modelling can be a useful tool for use in the interpretation of FRA data. This paper advances FRA interpretation through the development of physically representative transformer models. In the paper we focused on the End to End FRA test of the high voltage windings of a Dyn connected transformer. However, it could equally be applied to other vector group and FRA test configurations. The generic nature of the proposed model also enables the simultaneous fitting of the model to each of the three FRA test permutations.

The proposed transformer model was applied to the FRA data from a $1.3 \mathrm{MVA} 11 \mathrm{kV} / 430 \mathrm{~V}$ distribution transformer. The results in both magnitude and phase across frequencies up to $1 \mathrm{MHz}$ were very good. Since the model is based on physical parameters, the resulting parameter estimates were
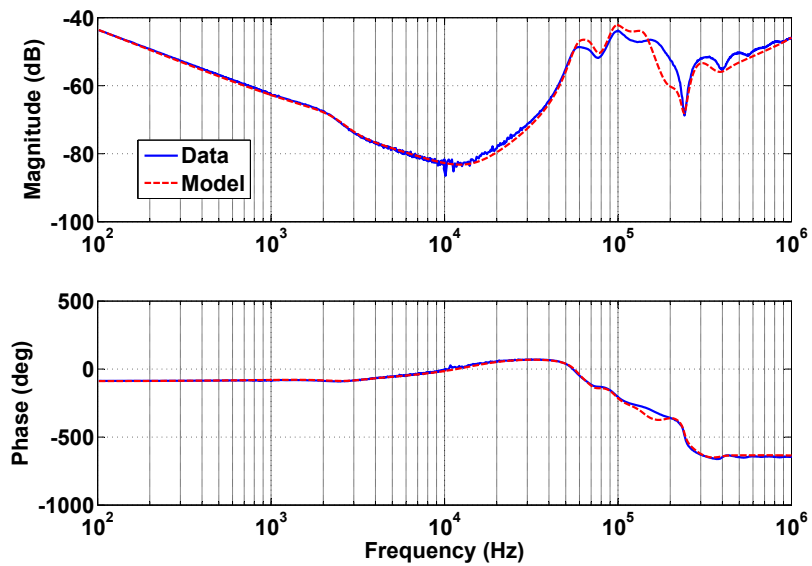

Figure 8. Estimated model and FRA data measured between the high voltage $\mathrm{C}$ and $\mathrm{B}$ terminals on a $1.3 \mathrm{MVA} 11 \mathrm{kV} / 430 \mathrm{~V}$ Dyn 1 transformer.

compared with the actual values with satisfactory results. These outcomes validate the modelling approach.

\section{REFERENCES}

[1] R. Heywood, M. Boltze, and S. Markalous, "Power transformer condition monitoring and life-cycle management," Euro TechCon, Stretton, United Kingdom, 2009.

[2] E. Bjerkan, "High frequency modeling of power transformers," $\mathrm{Ph} . \mathrm{D}$. dissertation, Mathematics and Electrical Engineering, May 2005.

[3] "Mechanical condition assessment of transformer windings using frequency response analysis (fra)," ELECTRA-CIGRE WG A2.26 Report, vol. 228, October 2006.

[4] J. H. Harlow, Ed., Electric Power Transformer Engineering, ser. The Electric Power Engineering Series. CRC Press, 2004.

[5] S. D. Mitchell and J. S. Welsh, "The influence of complex permeability on the broadband frequency response of a power transformer," Power Delivery, IEEE Transactions on, vol. PP, no. 99, pp. 1-1, 2009.

[6] E. C. Snelling, Soft Ferrites. Iliffe Books Ltd, 1969.

[7] P. Dowell, "Effects of eddy currents in transformer windings," Proc.IEE, vol. 113, pp. 1387-1394, 1966.

[8] J. Ferreira, "Improved analytical modeling of conductive losses in magnetic components," Power Electronics, IEEE Transactions on, vol. 9 , no. 1, pp. 127-131, Jan 1994.

[9] X. Nan and C. Sullivan, "An improved calculation of proximity-effect loss in high-frequency windings of round conductors," vol. 2, June 2003 , pp. 853-860 vol.2.

[10] N. Abeywickrama, Y. Serdyuk, and S. Gubanski, "High-frequency modeling of power transformers for use in frequency response analysis (fra)," Power Delivery, IEEE Transactions on, vol. 23, no. 4, pp. 20422049, Oct. 2008.

[11] C. Dervos, C. Paraskevas, P. Skafidas, and P. Vassiliou, "Dielectric characterization of power transformer oils as a diagnostic life prediction method," Electrical Insulation Magazine, IEEE, vol. 21, no. 1, pp. 1119, Jan.-Feb. 2005.

[12] "Mechanical condition assessment of transformer windings using frequency response analysis (fra)," ELECTRA-CIGRE WG A2.26 Report 342, vol. 237, April 2008.

[13] S. Mitchell, J. Welsh, and B. Phung, "Relating a distribution transformer's connection topology and the influence of inductive disparity to the observed frequency response," Universities Power Engineering Conference,2008. AUPEC'08. Australasian, 2008.

[14] R. A. Rohrer, Circuit Theory: An Introduction to the State Variable Approach. McGraw-Hill, 1970. 